\title{
Inteligencia de negocios y su incidencia en las organizaciones
}

\section{Business intelligence and its impact on organizations}

Bustamante, Mariana E.

Universidad Estatal del Sur de Manabí, Ecuador

Bustamante, César A.

Morales, David P.

Universidad Tecnológica Ecotec, Ecuador

Autor para correspondencia: cbustamante@ecotec.edu.ec

Fecha de recepción: 03 de Agosto de 2017 - Fecha de aceptación: 10 de Agosto de 2017

Resumen: Este artículo se centra en destacar la importancia en optimizar la gestión de la información en las organizaciones, como un recurso que les permita posicionarse en el entorno globalizado e híper competitivo actual, y tomar decisiones con menor grado de incertidumbre ante los diversos eventos presentados en los escenarios actuales. El Almacén "Mujer al Día" se dedica a la comercialización de ropa con diferentes líneas de accesorios complementándolo con un servicio personalizado. De acuerdo a los cambios constantes de la moda y a las exigencias cada vez mayores de los consumidores, requiere de diversas herramientas para captar y analizar los cambios propios de la dinámica que caracteriza la época actual, el gerente propietario y tomador de decisiones requiere de un acceso rápido y fácil a información útil y valiosa de la empresa. Se implementa un sistema de inteligencia de negocios en el área de marketing con herramientas que permiten generar el conocimiento necesario, a fin de aumentar el porcentaje de ventas mediante el diseño de estrategias de mercadeo. Por consiguiente, se realiza un estudio de mercado para conocer los gustos y preferencias de los clientes y competidores a través de encuestas; se aplicaron técnicas de recopilación de datos, tales como entrevista al propietario para conocer la situación actual de la empresa. Además, se realizan comparaciones de ingresos y egresos de los últimos meses para determinar las variaciones del volumen de ventas. Según estadísticas sobre preferencias de consumo, uno de los motivos por los cuales el negocio ha perdido su posicionamiento de mercado, fue por la escasa publicidad e insuficiente mercadería para abastecer la demanda, realizándose un análisis FODA permitiendo usar estos datos para el diseño de tácticas de producto, promoción, plaza y precio con el fin de ampliar la cobertura de mercado.

Palabras claves: inteligencia de negocios; ventaja competitiva; información; aplicaciones; soluciones

Abstract: This article focuses on the importance in optimizing the management of information in organizations as a resource that allows them to position themselves in today's globalized and better-funded environment, and make decisions with lower degree of uncertainty to events presented in the current scenarios. "Women's day" store is dedicated to the marketing of clothing with different lines of accessories complemented by a personalized service. According to the constant changes of fashion and demands ever-increasing consumer, requires different tools to capture and analyze the changes of dynamics that characterizes the current era, the owner and taker Manager of decisions requires quick and easy access to valuable and useful information of the 
company. Due to the wide range of software a business in the area of marketing with tools to generate the necessary knowledge, for to increase the percentage of sales through the design of marketing strategies. Therefore, is a market study to learn about the tastes and preferences of customers and competitors through surveys; collection of data, such as interview techniques were applied to the owner to know the current situation of the company? In addition, comparisons of income and expenditures for the past months are performed to determine variations in the volume of sales. According to statistics on consumer preferences, one of the reasons why the business has lost its market positioning, was by advertising scarce and insufficient goods to meet the demand, doing a SWOT analysis allowing to use these data for the design of product, promotion, and place and price tactics in order to expand market coverage.

Key words: business intelligence; competitive advantage; information; applications; solutions

\section{Introducción}

Este artículo se centra en destacar la importancia de los avances en las Tecnologías de Información, ofreciendo herramientas de gran capacidad que se han desarrollado como aplicaciones de soporte para la toma de decisiones, en diversos niveles de las organizaciones, generando conocimiento, base para la Inteligencia del Negocio. La gestión del Conocimiento, desarrolla las competencias centrales en las organizaciones.

Se presentan opiniones de connotados autores sobre la relevancia de la información. Se describen de manera muy general las herramientas que soportan la toma de decisiones, así como la ventaja estratégica de la Inteligencia del Negocio.

El almacén "Mujer al Día" es un local de ropa y calzado que nace en la ciudad de Jipijapa hace más de 23 años. Inicialmente fue administrada como una pequeña empresa familiar dirigida por el Sr. Yúber Rivera, al principio se enfocó en la venta de ropa para hombres, mujeres y niños. En poco tiempo se convirtió en uno de los mejores dentro del mercado, dándose a conocer por la variedad y calidad de productos que satisfacían las necesidades de los clientes.

A lo largo del tiempo el almacén dio paso a vender calzado, ya que había una gran cantidad de demanda insatisfecha, además de que ofrecen una gran variedad de ropa, lencería y demás accesorios como: carteras, cinturones y collares. Ésta fue reconocida por sus clientes debido a la excelente atención al mismo y la calidad e innovación de sus productos.

La empresa tuvo un crecimiento estable a lo largo de estos años, sin embargo, el ingreso de nuevos competidores terminó desplazándola hasta perder participación en el mercado, por ende, su desarrollo y el volumen de ventas ha disminuido. Actualmente, la empresa busca plantearse estrategias para crecer a nivel provincial en sus dos puntos iniciales de venta, considerando la situación del mercado que cada vez está más competitivo y sobretodo en locales de ropa. Para esto es indispensable tener una estrategia definida de marketing, apoyada por herramientas tecnológicas que permitan transformar la información en conocimiento asertivo para la toma de decisiones y estas permitan alcanzar los objetivos del estudio.

La información es un factor crítico para el éxito empresarial, la cual unido a un excelente plan de marketing; podrán complementarse y determinar las necesidades reales del mercado o de 
los clientes potenciales e identificar de una mejor manera comunicar el producto y servicio al cliente.

Hoy en día, las organizaciones manejan un flujo de información el cual era inimaginable apenas unos años atrás. Gracias a que nuestro mundo es ahora mucho más instrumentado, la capacidad de recolectar datos es impresionante. Es aquí donde entra una de las herramientas más mencionadas últimamente en el área de negocios: Inteligencia de Negocios. Se refiere directamente a la práctica y al conjunto de herramientas tecnológicas que pueden ayudar a las empresas a adquirir un mejor entendimiento de ellas mismas. Esto gracias a la capacidad de explotar su información, con la intención de poder manipularlos de una manera más sencilla y entender el porqué de nuestro desempeño o mejor aún, plantear escenarios a futuro, lo cual nos ayudará a tomar mejores decisiones.

En base a lo anteriormente descrito el Almacén Mujer al Día, que es una empresa mediana, recurre a la necesidad de herramientas sumamente especializadas, operando por un conjunto de reglas y prácticas mucho más equitativas a las de sus rivales, encontrando soluciones de inteligencia de negocios con tecnológica abierta y enfocadas al usuario final, que contemplan todo el poder del análisis, visualización y planeación de las soluciones de inteligencia de negocios.

Con ventajas tan grandes como el análisis de negocios "en memoria", que permite un trabajo increíblemente rápido y con datos mucho más actualizados y el manejo de datos en un ambiente flexible, para después realizar un elaborado plan de marketing con el fin de establecer nuevos canales de introducción al mercado, el mismo que la organización incursiona ya hace 23 años y que con el paso del tiempo se ha convertido en el lugar idóneo para adquirir prendas de vestir, calzado o algún artículo relacionado con la línea de su competencia.

De igual manera con el transcurso del tiempo hace necesario que se apliquen nuevas formas de atraer clientes por lo que se decide que la estrategia adecuada para lograrlo es aplicar técnicas profesionales actuales que permitan alcanzar el objetivo propuesto.

Se aplican técnicas avanzadas de marketing basadas en el conocimiento generado de las herramientas tecnológicas de inteligencia de negocios; con el fin de elaborar un plan de reposicionamiento para el Almacén "Mujer al Día", en el cual se busca convertir al local en un próspero negocio, alcanzando niveles de venta óptimos de los productos que oferta, es necesario refrescar la imagen de la empresa para cambiar las percepciones negativas del servicio al cliente y lograr un crecimiento de la empresa en el largo plazo, pero antes es necesario elaborar y cumplir ciertas reglas básicas establecidas dentro de los parámetros comerciales actuales que son elaborados con la información obtenida en el Sondeo Rápido de Mercado sobre el segmento donde va a ir destinado el producto, poniendo énfasis principalmente en las debilidades encontradas, fortaleciendo y aprovechando las oportunidades que el mismo estudio brinde.

Entonces, el objetivo central de esta investigación es desarrollar un plan de marketing y el diseño de estrategias para incrementar el porcentaje de ventas, realizando investigación de mercado para determinar los gustos y preferencias de las tendencias de la moda actual mediante 
el uso de instrumentos y aplicaciones de Tecnologías de Información (TI), los cuales están enfocados en la creación del conocimiento, a través de los datos existentes en la organización.

\section{Referentes Teóricos}

La literatura recoge numerosos trabajos de investigación que han tratado de dar respuesta al reto del cambio. En los últimos años, se asiste a la aparición de nuevos modelos y teorías que pretenden facilitar la comprensión y dirección de las grandes y complejas organizaciones actuales (Teece, Pisano \& Shuen, \& Rajagopalan \& Rajagop, 1997)

La mayoría de estos modelos señalan diversas herramientas a aplicar y características que facilitan el proceso de cambio, como la capacidad para innovar y aprender, la calidad total y la mejora continua, la importancia de los recursos humanos, la reingeniería, el outsourcing, o la orientación al cliente, por mencionar sólo algunos de los factores más citados. Una observación de esta relación permite detectar dos grandes temas de interés: el cambio o enfoque dinámico de los problemas y el desarrollo de los recursos y capacidades de la organización. Esto supone, en otras palabras, una convergencia entre el interés por el mercado - manteniendo el mayor grado de ajuste posible con su evolución - y el interés por los recursos y capacidades internas de la empresa. Es decir, el cambio implica el reto, no exento de paradoja, de renovar la organización sin destruir las capacidades básicas que generan ventajas competitivas (Hamel \& Prahalad, 1998; Fernández y Fernández, 1998).

En la obra, La naturaleza del trabajo directivo, (Mintzberg, 1992) distinguía tres tipos de roles directivos: interpersonales, informativos y decisionales. En un primer momento, los sistemas de información iniciales apoyaban principalmente los roles informativos. Sin embargo, durante los últimos años se han desarrollado sistemas de información que apoyan los tres roles.

En la actualidad, existen diferentes sistemas de información de apoyo a la gestión que tienen como propósito servir de apoyo en las distintas tareas que componen el proceso de toma de decisiones.

"No es suficiente tener una mente buena, lo principal es usarla bien." (Descartes) "No se puede controlar lo que no se puede medir" (Marco) "La información es una fuente de aprendizaje. Pero a menos que esta esté organizada, procesada y disponible para la persona adecuada en un formato para la toma de decisiones, esta es una carga, no un beneficio" (Polard) hacia el concepto de Inteligencia de Negocios hace algo más de 50 años, en octubre de 1958, fue publicado un artículo en la revista IBM Journal donde H.P.

Luhn un investigador de la empresa International Business Machines Corp. (IBM), pionero en las ciencias de la información utilizó el término "Business Intelligence System" para referirse a un sistema automático que acepta información en su formato original, disemina los datos adecuada y rápidamente a los lugares correctos.” Según (Luhn, 1958) para cumplir con estos objetivos era necesaria la aplicación de diferentes técnicas como en su momento lo fueron: Auto abstracción de documentos, auto codificación de documentos, creación y actualización automática de perfiles de usuarios. Sin embargo, estas técnicas para el procesamiento de los datos principalmente son de carácter estadístico y no son eficaces si no se cuenta con facilidades 
en los sistemas de comunicación, así como en los medios de entrada-salida, desde el punto de vista administrativo un sistema inteligente para los negocios provee la facilidad en la comunicación que permite la conducción de un negocio, donde inteligencia es capacidad de entender las interrelaciones de hechos pasados de tal manera que estos guíen las acciones hacia una meta deseada. Según su trabajo, el objetivo del sistema es "proporcionar la información adecuada para soportar actividades específicas realizadas por individuos, grupos, departamentos, divisiones o aun unidades más grandes... estas actividades se deben realizar rápida y eficientemente." Las investigaciones de Luhn pretendían el desarrollo de un sistema automatizado de análisis de texto que permitiese gestionar los documentos que se generan en una empresa, el cual mediante un procesamiento y un sistema de almacenamiento eficiente permitiese a los empleados realizar búsquedas sobre temas específicos y el sistema remitiría los documentos adecuados a los usuarios, de acuerdo a unos perfiles establecidos.

Planteó que "la mejor forma de analizar el entorno no es tratar de entenderlo como una colección de otros sistemas y organizaciones, sino tratarlo como información a la cual la empresa debe acceder mediante actividades de investigación". Asimismo, afirmó que "no son los proveedores ni los clientes en sí mismos los que cuentan, sino la información disponible para la empresa acerca de sus metas, intereses, condiciones bajo las cuales se relacionan con la empresa y muchos otros aspectos de su comportamiento, los que verdaderamente ayudan a identificar sus características y expectativas para con la organización".

(Wiener, Ashby, \& Beer, 1950; 1957; 1959) Acerca de la forma como se regulan los sistemas y particularmente de los efectos que tiene la información en la regulación de los mismos. Estos postulados, pese a que no se desarrollaron a la par con el concepto de Inteligencia de Negocios, tienen una estrecha relación.

(Cardona, 2005) Adjudica la creación del término Inteligencia de Negocios a Richard Green, quien en el año 1966 lo definió como "la información procesada de interés para la administración acerca del presente y futuro del entorno en el cual el negocio debe operar”.

(Negash \& Gray, 2003): "Los sistemas de inteligencia de negocios combinan la obtención y almacenamiento de datos, así como la gestión del conocimiento con herramientas analíticas que presentan información compleja y competitiva a los planificadores y decisores". En esta definición se encuentra de forma implícita la idea de que estos sistemas proporcionan información sobre la que se puede actuar, distribuida en el momento y lugar adecuado, así como en el formato correcto para asistir a los decisores.

Dentro del mundo de los negocios y, de las organizaciones en general, la "Inteligencia de Negocios", es un concepto respaldado por una nueva manera de hacer las cosas, posible, gracias a los avances de los Sistemas de Información y de las Tecnologías de Información.

\section{Metodología}

Esta investigación hace un análisis descriptivo y estadístico a través de los resultados obtenidos del instrumento científico (encuesta), teniendo como objetivo tabular los datos de las 
encuestas, sistematizarlos, interpretarlos, graficarlos y de acuerdo a ello realizar las conclusiones respectivas (Rodríguez, Pierdant, \& Rodríguez, 2016).

El estudio se encuentra dentro del paradigma cuantitativo (Ojeda, 1998) examinando datos de manera científica especialmente la forma numérica con ayuda del campo estadístico.

\section{Población y Muestra}

Población: Indica el INEC (Instituto Nacional de Estadísticas y Censos) que en la zona urbana hay 71.083 habitantes.

Muestra: Según el cálculo de la muestra se determinó hacer una encuesta a 352 personas de la ciudad de Jipijapa. (Se demuestra con la siguiente formula).

$$
\begin{gathered}
n=\frac{(Z)^{2}\left(P^{*} Q\right)(M)}{(e)^{2}(\mathrm{~N})+(Z)^{2}\left(P^{*} Q\right)} \\
n=\frac{(1,88)^{2}(0,36)(71.083)}{(0,06)^{2}(71,083)+(1,88)^{2}(0,36)} \\
n=\frac{(3,53)(0,36)(71.083)}{(0,36)(71,083)+(3,53)(0,36)} \\
n=\frac{90.332}{255,89+1,27} \\
n=\frac{90.332}{257,16} \\
n=352 \text { habitantes (encuestas) }
\end{gathered}
$$

\section{Resultados}

El estudio de mercado fue aplicado a la ciudadanía Jipijapense, del cual se obtuvieron los siguientes resultados: Se deduce que, al momento de efectuar el perfil del cliente, resultará más factible describirlos en las edades de 14 - 40 años, consecuentemente debido a que fueron los que principalmente participaron en la encuesta, por consiguiente, con estos se tendrá una ventaja para definir estrategias que permitan lograr el objetivo. Además, se conoce que la mayoría de la población Jipijapense (44\%) no tiene conocimiento sobre el almacén "Mujer al Día", por ende, la aplicación del Plan de Marketing ayudará a reposicionar en el mercado sus servicios para que las personas conozcan este local e incremente el número de clientes.

La gran mayoría de consumidores se encuentran satisfechos con la variedad de productos, pese a este hecho positivo, se analizarán mejoras para las personas inconformes que constituyen el $18 \%$, observando y examinando las falencias que ofrece el servicio.

Se afirma que el cliente toma mucho en cuenta el precio y la calidad del producto antes de comprarlo, además, lo que más lo atrae es la buena atención de los empleados y propietario. Por lo tanto, es necesario implementar estrategias de servicio al cliente, asimismo se evaluó los dos primeros factores (precios y calidad) y se concluyó conjuntamente con las respuestas de los 
consumidores que este almacén si posee lo que el cliente busca para preferir nuestras prendas de vestir. Se detectó que el mejor medio de comunicación para promocionar y hacer publicidad se encuentra en las redes sociales como Instagram y Facebook, pues la colectividad de Jipijapenses gozan de este servicio cómodo y gratuito de marketing, a más de algunas personas mayores de edad que prefieren que se de publicidad por televisión o radio.

La gran mayoría de encuestados consideran que la atención que brindan es buena, consecuentemente la empresa se encuentra en un nivel medio, no obstante, existen personas que reconocen el esfuerzo de los emprendedores y consideran su excelencia al alto nivel de cortesía. También manifestaron que el local está en una buena ubicación, ya que es céntrico y por lo tanto hay mayor movilización de personas.

\section{Perfil del cliente}

Según el respectivo análisis del cliente, las personas del género masculino y femenino de las edades de 14 - 40 adquieren ropa en el almacén "Mujer al Día", en el nivel económico son los de clase media, hacen sus compras cada mes (53\%), el nivel de ingresos disponible es medio, según los datos recopilados en la encuesta las personas toman mucho en cuenta los precios calidad y marca de los productos antes de hacer su compra y los gustos o preferencia del cliente según el orden jerárquico fueron: Pantalones, shorts, vestidos, camisas, camisetas, pantalonetas y suéteres.

Simón Andrade, autor del libro "Diccionario de Economía", proporciona la siguiente definición de demanda: "Es la cantidad de bienes o servicios que el comprador o consumidor está dispuesto a adquirir a un precio dado y en un lugar establecido, con cuyo uso pueda satisfacer parcial o totalmente sus necesidades particulares o pueda tener acceso a su utilidad intrínseca".

Tabla\#1: Perfil del cliente

\begin{tabular}{|l|l|}
\hline ¿Quiénes serán tus clientes? & Hombres y Mujeres (100\%). \\
\hline Genero & Masculino y Femenino (100\%). \\
\hline Número de Clientes & $14-40$ (100\%) \\
\hline Nivel de ingresos & Medio. \\
\hline Frecuencia de compra del producto & Cada mes (53\%). \\
\hline $\begin{array}{l}\text { ¿Qué toman en cuenta las personas para } \\
\text { hacer una compra? }\end{array}$ & $\begin{array}{l}\text { El precio, calidad y la marca de los } \\
\text { productos. }\end{array}$ \\
\hline Gustos y preferencia del Cliente & $\begin{array}{l}\text { Pantalones, shorts, vestidos, } \\
\text { camisas, camisetas, pantalonetas y } \\
\text { suéters. }\end{array}$ \\
\hline
\end{tabular}

Fuente: Autoría propia 


\section{Perfil de la competencia}

Gracias al análisis de la competencia que se realizó, se pudo determinar que D’Compras, Novedades Daniella, Barathon y el almacén "La Bahía” son una competencia ya que ofrecen el mismo producto, tienen un nivel de aceptación alto debido a que diariamente muchas personas recurren a estos comerciales, el tipo de cliente al que ellos atienden es al de clase alta, media y baja, las razones por las cuales las personas los eligen es por la buena localización en la que ellos se encuentran ya que es en el centro de la ciudad, también por la gran variedad de productos que ofrecen, el precio al que ofrecen sus productos es de aceptación por los clientes.

Con lo analizado se pudo concluir que, lo positivo de la competencia se debe a la buena localidad que tienen y a la buena calidad de sus productos, nosotros para poder tener una buena demanda debemos poner nuestro local en el centro de la ciudad ya que esto nos permitirá poder tener mejor acogida al momento de ofrecer nuestro producto también debemos ofrecer un producto de calidad y precios accesibles.

Estrategia: K. J. Halten (1987): “Es el proceso a través del cual una organización formula objetivos, y está dirigido a la obtención de los mismos. Estrategia es el medio, la vía, es el cómo para la obtención de los objetivos de la organización. Es el arte de entremezclar el análisis interno y la sabiduría utilizada por los dirigentes para crear valores de los recursos y habilidades que ellos controlan. Para diseñar una estrategia exitosa hay dos claves; hacer lo que hago bien y escoger los competidores que puedo derrotar. Análisis y acción están integrados en la dirección estratégica".

Tabla\#2: Análisis de la competencia

\begin{tabular}{|c|c|c|c|c|c|c|c|}
\hline \multicolumn{8}{|c|}{$\begin{array}{l}\text { ANÁLISIS DE LA COMPETENCIA } \\
\text { PERFIL DEL COMPETIDOR }\end{array}$} \\
\hline \multirow{2}{*}{$\begin{array}{l}\text { EMPRESAS } \\
\text { COMPETIDORAS }\end{array}$} & \multicolumn{3}{|c|}{ NIVEL DE ACEPTACIÓN } & \multirow{2}{*}{$\begin{array}{l}\text { TIPO DE } \\
\text { CLIENTE } \\
\text { QUE } \\
\text { ATIENDEN }\end{array}$} & \multirow{2}{*}{$\begin{array}{l}\text { ¿POR } \\
\text { QUÉ } \\
\text { RAZÓN } \\
\text { LO } \\
\text { EUGEN? }\end{array}$} & \multirow{2}{*}{$\begin{array}{l}\text { ¿DÓNDE SE } \\
\text { COMERCIAUZA } \\
\text { LOS } \\
\text { PRODUCTOS? }\end{array}$} & \multirow{2}{*}{$\begin{array}{l}\text { ¿EN QUE SE } \\
\text { DIFERENCIA } \\
\text { DE LOS } \\
\text { DEMAS } \\
\text { LOCALES? }\end{array}$} \\
\hline & ALTA & REGULAR & BAIA & & & & \\
\hline D'Compras & $X$ & & & $\begin{array}{l}\text { Clase } \\
\text { media y } \\
\text { baja. }\end{array}$ & $\begin{array}{l}\text { Buen } \\
\text { Servicio. }\end{array}$ & Jipijapa & Variedad. \\
\hline $\begin{array}{l}\text { Novedades } \\
\text { Daniella }\end{array}$ & & $X$ & & $\begin{array}{l}\text { Clase alta } \\
\text { y media. }\end{array}$ & Precio. & Jipijapa. & Calidad. \\
\hline Barathon & $X$ & & & $\begin{array}{l}\text { Clase } \\
\text { media y } \\
\text { baja. }\end{array}$ & $\begin{array}{l}\text { Buen } \\
\text { Servicio. }\end{array}$ & Jipijapa. & Promociones. \\
\hline $\begin{array}{l}\text { Almacén “La } \\
\text { Bahía” }\end{array}$ & & $\mathbf{X}$ & & $\begin{array}{l}\text { Clase } \\
\text { media y } \\
\text { baja. }\end{array}$ & Precio. & Jipijapa. & $\begin{array}{l}\text { Buen Servicio } \\
\text { y Precio. }\end{array}$ \\
\hline
\end{tabular}

Fuente: Autoría propia 


\section{Análisis situacional}

El almacén "Mujer al Día" ha mantenido un crecimiento sostenible en lo últimos años, sin embargo, la entrada de nuevos competidores y la expansión de la categoría de ropa ha provocado que este crecimiento sea lento, lo que preocupa por que así el negocio estaría perdiendo y podría llegar a quebrar.

La competencia ha logrado hacer crecer la categoría mediante la introducción de nuevos productos e innovando en el ambiente de los locales. Los vendedores informales de ropa han migrado a formatos más amigables para el comprador; donde pueden pasar un momento agradable, mientras buscan la prenda que necesitan.

El almacén no ha tenido innovación en cuanto a carteras, cinturones y calzado. La última tendencia fueron las carteras imitación de "Nicole Lee", cinturones gruesos de diversos colores y zapatos deportivos para hombres, mujeres y niños. En cuanto a la ropa de mujer esta actualizado con las últimas tendencias.

\section{Análisis FODA}

Este análisis permitió identificar puntos claves para establecer el plan de mercadeo del almacén "Mujer al Día".

\section{Fortalezas}

- Es que en todo momento y lugar la gente se viste.

- Una empresa compacta en infraestructura física y recurso humano.

- Sostenibilidad en el tiempo.

- Variedad de productos.

- $\quad$ Empresa con más de 23 años en el mercado.

Oportunidades

- Alcanzar el segmento joven a través de los medios de comunicación y nuevos productos que capten la atención del público.

- Entablar líneas de negocios con proveedores internos y externos que ubicarán la marca en diferentes puntos geográficos.

- Crecimiento de categoría por la introducción de nuevos productos.

\section{Debilidades}

- El inicio de las actividades de la empresa.

- Posicionamiento del producto al inicio, que luego se convertirá en una fortaleza.

- No tiene el reconocimiento de tener ropa de buena calidad.

\section{Amenazas}

- Baja de venta debido a la inestabilidad política y económica.

- Desastres que afecten la infraestructura. 
- Crecimiento de empresas especializadas en Ventas/Marketing, dirigidas por profesionales con estudios del exterior.

\section{Conclusiones del análisis FODA}

Fortalezas > Debilidades: El almacén "Mujer al Día" debe establecer una estrategia de publicidad para que vuelva a ser reconocido por su alta innovación y calidez de productos. Es necesario implementar un plan de marketing estratégico, acompañando de una campaña reposicionamiento del negocio.

Fortalezas > Oportunidades: El almacén "Mujer al Día” debe aprovechar la oportunidad del crecimiento de la categoría por medio del desarrollo de nuevas líneas de productos que se adapten a las nuevas tendencias del mercado. Esto permitirá a la empresa mantener en la mente de los clientes su reconocimiento de un negocio de años en el mercado, pero que se mantiene en constante innovación.

Amenazas > Oportunidades: Debido a la inestabilidad de política y económica del país se debe ser cauteloso respecto a la inversión en nuevos proyectos. Además, es importante llevar un control sobre los ingresos y gastos de la empresa para poder reaccionar frente a desastres que afectan la infraestructura.

\section{Análisis Mix}

“El marketing mix es uno de los elementos clásicos del marketing, es un término creado por McCarthy en 1960, el cual se utiliza para englobar a sus cuatro componentes básicos: producto, precio, distribución y comunicación. Estas cuatro variables también son conocidas como las 4Ps por su acepción anglosajona (product, price, place y promotion)'”. Define Roberto Espinoza.

\section{- $\quad$ Producto}

"El producto es la variable por excelencia del marketing mix ya que engloba tanto a los bienes como a los servicios que comercializa una empresa. Es el medio por el cual se satisfacen las necesidades de los consumidores". Roberto Espinoza.

En el almacén se ofrece una variedad de ropa, calzado y accesorios, para el análisis se necesitarán los siguientes puntos relacionados al producto, como ejemplo: la diferenciación de estos productos con los de la competencia, marcas, modelos y empaques; modificación y eliminación del producto; planificación de nuevos modelos.

En este caso, los productos del almacén son múltiples, tanto como sus prendas de vestir, calzados y accesorios, además, se diferencian de la competencia por ser artículos de calidad y variados.

\section{- $\quad$ Precio}


"El precio es la variable del marketing mix por la cual entran los ingresos de una empresa. Antes de fijar los precios de nuestros productos debemos estudiar ciertos aspectos como el consumidor, mercado, costes, competencia, etc. ”. Roberto Espinoza.

Para el análisis del valor de este bien se tomará en cuenta los siguientes componentes; el costo del producto o servicio, el nivel de la competencia, la demanda del producto o servicio, la disponibilidad de los consumidores. El almacén "Mujer al Día" goza un precio de venta al público desde $\$ 14,00$ hasta $\$ 32,00$ para blusas, pantalones, shorts, vestidos, el calzado es desde $\$ 23,00$ hasta $\$ 45,00$ dependiendo de los zapatos que compre y los accesorios cuestan entre $\$ 18,00$ a $\$ 25,00$.

\section{Situación financiera}

El almacén "Mujer al Día” busca reposicionar su servicio en la ciudadanía Jipijapense, por lo que se invertirá en publicidad.

Con la finalidad de conocer las ventas de distintos meses, estipular la situación financiera en la que se encuentra el negocio y si es posible aplicar el plan de marketing, se evaluaron los periodos consecutivos desde agosto-octubre. Este estudio se realizó por cada semana del mes, en donde se estudiaron las disminuciones e incrementaciones de ventas, colectivamente con los gastos incurridos.

\section{Ingresos}

Los ingresos del almacén "Mujer al Día" que provienen de las ventas de los artículos que los clientes adquieren diariamente. Se consideran los ingresos de meses pasados para hacer la pertinente identificación de la aplicación viable-financiera del Plan de Marketing.

Tabla \# 3: Ingresos Agosto-septiembre-octubre 2015

\begin{tabular}{ccccccc}
\hline Semanas & Agosto & $\%$ & Septiembre & $\%$ & Octubre & $\%$ \\
\hline 1 & $\$ 150,00$ & $22 \%$ & $\$ 190,00$ & $27 \%$ & $\$ 220,00$ & $28 \%$ \\
2 & 280,00 & $24 \%$ & 160,00 & $25 \%$ & 170,00 & $20 \%$ \\
3 & 210,00 & $26 \%$ & 195,00 & $26 \%$ & 250,00 & $23 \%$ \\
4 & 300,00 & $28 \%$ & 180,00 & $22 \%$ & 320,00 & $29 \%$ \\
TOTALES: & $\$ 755,00$ & $100 \%$ & $\$ 725,00$ & $100 \%$ & $\$ 960,00$ & $100 \%$ \\
\hline
\end{tabular}

Fuente: Autoría propia

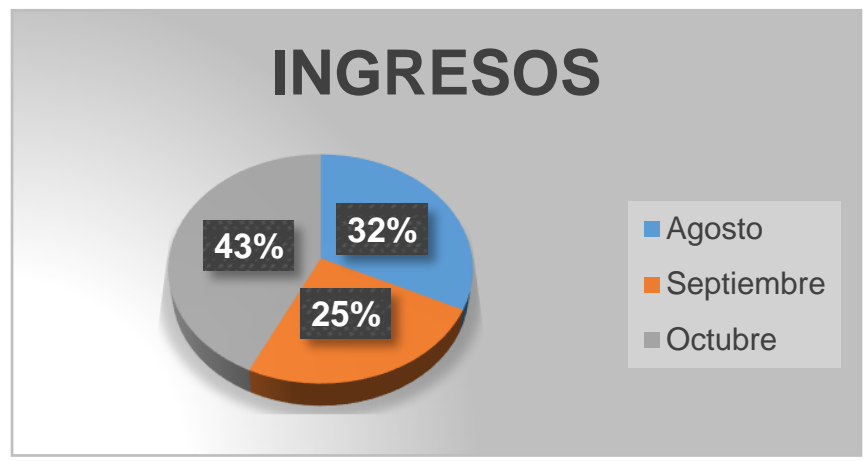




\section{Grafico \#1: Ingresos \\ Fuente: Autoría propia}

Las actividades que desarrollo el local en los meses de agosto, septiembre y octubre fueron las siguientes: En la primera semana del mes de agosto se generó ingresos de \$150,00 y representa el $22 \%$ de sus ventas semanales. En la segunda semana fue de $\$ 280,00$ que equivale al $24 \%$; la tercera semana fue de $\$ 210,00$ que es igual al $26 \%$. Finalmente, la cuarta semana fue de $\$ 300,00$ con un valor porcentual de $28 \%$. Almacén “Mujer al Día” tuvo mejores ventas en la cuarta semana, debido a sus estrategias para atraer sus clientes como lo es la buena atención al mismo.

En la primera semana del mes de septiembre se concibió ingresos de \$190,00 que semeja el 27\% de sus ventas semanales. En la segunda semana fue de \$160,00 que es paralelo al 25\%; la tercera semana fue de $\$ 195,00$ y representa el $26 \%$. En último lugar la cuarta semana fue de $\$ 180,00$ con un valor porcentual de $22 \%$, tuvo mejores ventas en tercera semana.

En la primera semana del mes de octubre se forjó ingresos de $\$ 220,00$ que es igual al $28 \%$ de sus ventas semanales. En la segunda semana fue de $\$ 170,00$ que equivale al 20\%; la tercera semana fue de $\$ 250,00$ que refleja el $23 \%$. Posteriormente la cuarta semana fue de $\$ 320,00$ que representa el $29 \%$, tuvo mejores ventas en la cuarta semana.

\section{Gastos}

Los gastos que incurre mensualmente el almacén "Mujer al Día” son los siguientes:

\begin{tabular}{cc}
\multicolumn{2}{c}{ Tabla\#4: Gastos } \\
\hline \multicolumn{2}{c}{ Almacén "Mujer al Día" } \\
\hline Servicios Básicos & $\$ 60,00$ \\
Arriendo & 120,00 \\
Sueldos & 180,00 \\
TOTALES. & $\$ 360,00$ \\
\hline
\end{tabular}

Fuente: Autoría propia

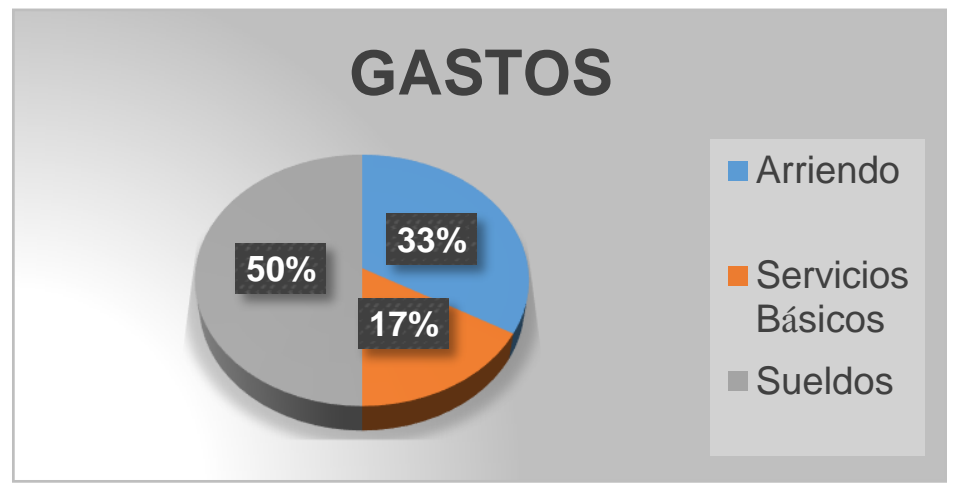

Grafico \#2: Gastos Fuente: Autoría propia 
Durante el análisis de los meses de agosto-octubre, se pudo detectar que el almacén "Mujer al Día" posee un promedio mensual de $\$ 2.440,00$ y gastos mensuales de $\$ 360,00$ por lo tanto durante el mes está obteniendo una rentabilidad de \$2.080,00.

\section{- $\quad$ Plaza}

Almacén "Mujer al Día" se encuentra ubicado en las calles Sucre y Santisteban del Cantón Jipijapa, su canal de distribución es directo pues venden sus productos directamente a los clientes en el local.

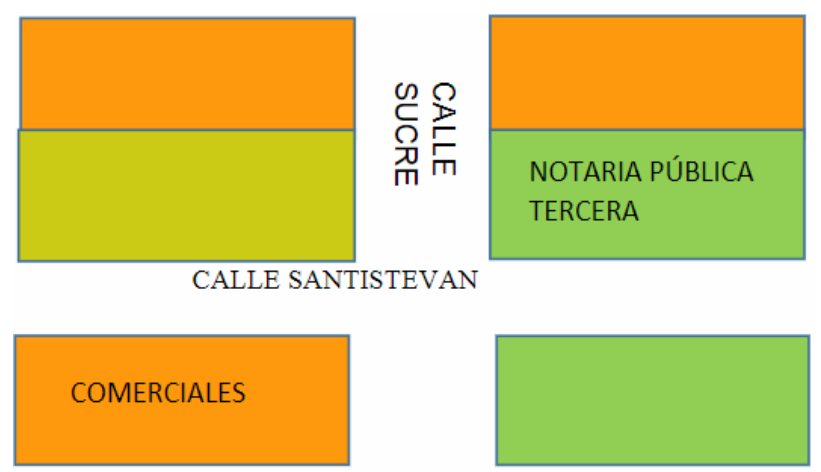

Grafico \#3: Croquis del lugar de ubicación

\section{- $\quad$ Promoción}

El segmento identificado fueron los jóvenes y adultos de 14-40 años, para llegar al grupo objetivo se utilizó como medio principal internet, este se analizó desde el estudio de mercado en donde los encuestados mayormente con un $73 \%$ lo eligieron como medio por el cual les gustaría conocer el producto. Se busca reposicionar al almacén "Mujer al Día" dando a conocer la variedad de artículos y servicio que este brinda a sus clientes.

\section{Conclusión del programa Marketing Mix}

La mezcla del marketing mix es un término utilizado para referirse a un grupo de herramientas y factores que poseen las empresas para su crecimiento continuo. Además, es un instrumento que incluye una combinación de las 4Ps; Precio, Producto, Promoción y Plaza.

Se efectuó un plan de comunicación para interactuar con los clientes del almacén "Mujer al Día" mediante estrategias diseñadas desde la recopilación de datos en las 4Ps, en donde se plantearon tácticas para hacer del negocio un lugar conocido por su variedad de artículos y buena atención al cliente.

Por ende, la aplicación del programa de marketing mix permite que el almacén "Mujer al Día" adquiera una mejor participación en el mercado y se mejore su rentabilidad a largo plazo corrigiendo las falencias internas y externas.

\section{Conclusión}


Se identificaron y analizaron los gustos y preferencias de los clientes; así como la calidad y servicio al cliente. Una parte de este conocimiento provino por medio de las encuestas, se recopiló información acerca del negocio, incluyendo el marketing mix (precio, producto, promoción y plaza) para proceder al planteamiento de estrategias; y el otro tanto por el conocimiento que residía en los almacenes de datos, y mediante el uso de herramientas tecnológicas de búsqueda de patrones se las pudo detectar.

Adicionalmente se estableció a la competencia y las estrategias que implementan. Sin embargo, se conoció que la mayoría apuesta "la buena atención al cliente" como estrategia más usada que permite la captación de consumidores, no obstante, por desconocimiento no aplican metodologías que permitan lograr el mismo objetivo.

En este escenario, es importante evaluar y analizar la utilización de este tipo de tecnologías en el cantón Jipijapa, para entender su dinámica y así mismo proponer mejoras, mediante el desarrollo de estudio se identificó el perfil del cliente y competencia, además, se analizaron los problemas que rodean el entorno del local, de esta manera se identificaron las oportunidades y debilidades del negocio para conocer la percepción de los consumidores tanto del servicio que brinda y el ambiente que ofrece.

Colectivamente, después de recolectar información de los estudios, se diseñaron estrategias de comunicación con el cliente y se planteó un plan de acción que permita cumplir con el objetivo, además, con la aplicación se logrará incrementar el porcentaje de ventas a largo plazo.

Actualmente nos encontramos y hacemos parte de una revolución tecnológica que se mueve en torno a las tecnologías de la información y comunicación. Como consecuencia, se habla de un cambio paradigmático el cual ha sido denominado "Sociedad de la información y el conocimiento". Un campo de afectación particular al respecto son los sistemas de soporte a la dirección y a las decisiones, dentro del cual los sistemas de inteligencia de negocios promueven la cooperación entre la tecnología y la gestión, basándonos en esta simbiosis se diseñó un plan de marketing en el almacén "Mujer al Día" para reposicionar su servicio en el mercado mediante la aplicación de investigaciones del micro entorno, en conjunto, se plantearon estrategias de marketing y publicidad.

La Inteligencia de Negocios o Business Intelligence es un conjunto de técnicas y herramientas tanto de gestión empresarial como de aplicación tecnológica, que permiten a partir de la formulación estratégica y teniendo como objetivo dar soporte a los procesos de planeación y control en las organizaciones, la extracción e integración de los datos, que son generados como resultado de la operación de las diferentes áreas funcionales en una organización, su posterior procesamiento y distribución en forma de información.

Luego de la evaluación mensual económica, se concluye que para el almacén "Mujer al Día” es necesario aplicar el proyecto propuesto pues con él logrará reposicionar su servicio y como una opción de preferencia para los clientes Jipijapenses. 
En último lugar, el estudio financiero expresa que es factible aplicar el plan de marketing pues la inversión es asequible monetariamente y resulta rentable la aplicación del plan estratégico para reposicionar al almacén "Mujer al Día” en el mercado.

\section{Bibliografía}

Cardona. (2005). Inteligencia De Negocios. Descartes, R. (S.F.). Tesis De Maestría Facultad De Ciencias Económicas.

Dill. (1958). La Importancia De La Seguridad Y Posesión De Los Datos.

Luhn. (1958). Business Intelligence System. Marco, T. D. (S.F.). Tesis De Maestría Facultad De Ciencias Económicas.

Mintzberg, H. (1992). La Gestión Organizacional Y La Toma De Decisiones. Negash, \& Gray. (2003). Inteligencia De Negocios.

Innova Research Journal 2017, Vol 2, No. 8, 159-173.Issn 2477-9024revistamensualde La UIDE Extensión Guayaquil

Rajagopalan \& Rajagop. (1997). La Importancia De La Inteligencia De Negocios Aplicada A Empresas Medianas. Wiener, Ashby, \& Beer. (1950; 1957; 1959). La Importancia De La Seguridad Y Posesión De Los Datos. Tesis De Grado (Espol) 2009, María Cristina Alvarado Enderica Y Francisco José Castro Vargas. Datos e Información Del Almacén "Mujer Al Día".

\section{Web Grafía}

http://robertoespinosa.es/2014/05/06/marketing-mix-las-4ps-2/

http://dspace.ups.edu.ec/bitstream/123456789/1526/13/UPS-GT000243.pdf

http://www.uovirtual.com.mx/moodle/lecturas/metoprot/10.pdf

http://www.marketing-xxi.com/concepto-de-investigacion-de-mercados-23.htm

http://www.marketing-xxi.com/el-plan-de-marketing-en-la-empresa-132.htm

http://definicion.de/rentabilidad/\#ixzz3tZY064Su

http://acualonit.overblog.com/las-4-p-preci-producto-plaza-y-promocion

http://www.definicionabc.com/ciencia/metodologia.php

Rafael Muñiz, http://www.marketing-xxi.com/etapas-del-plan-de-marketing-136.htm 